\title{
Covert orienting attention in Parkinson's disease
}

\author{
Tatsuo Yamada, Mutsuo Izyuuinn, Michael Schulzer, Keizo Hirayama
}

\begin{abstract}
We examined covert orienting attention in twenty patients with Parkinson's disease using Posner's reaction time (RT) method. Patients were divided according to the grade of severity (Hoehn and Yahr), the P1 group was grade I-II and P2 group grade II-IV. Each group of patients was compared with an equal number of age-matched controls. In controls, and in the less disabled younger P1 group, a significant RT difference was shown between "valid" and "crossed" conditions, that is, when the cue and target appeared in the same square or in equivalent squares in opposite visual fields. In the P2 group, however, there was no advantage of the "valid" over "crossed" conditions. Furthermore, the RT difference seen in controls between "valid" and "towards the fovea" or "away from the fovea" (conditions where cue and target were in adjacent squares) disappeared in the Parkinsonian groups. These results suggest that the covert shift of attention from initial focus to the cue is very weak, or does not occur in more disabled Parkinsonian patients, resulting in only a shift of attention to the target. In considering the elementary mental operations involved in covert orienting attention, this deficit may be attributable to a disturbance of moving attention.
\end{abstract}

Apart from generalised intellectual deterioration, various cognitive dysfunctions have been reported in patients with Parkinson's disease (PD). There have, however, been few reports on the attention and arousal deficits, such as diminished vigilance ${ }^{12}$ and visual neglect, ${ }^{3}$ which may underly cognitive dysfunctions. Using reaction time (RT) methods, certain aspects of attention and arousal can be evaluated. One such paradigm, proposed by Posner, ${ }^{4}$ is covert orienting of attention in the visual field. Facilitation of processing efficiency occurs when a cue is given at the same location as the target (valid condition) in comparison to other locations (invalid conditions). In the "valid" condition, the subject must orient their attention to a cue and then engage the target, while in "invalid" conditions, the subject must first disengage attention from the cued location, move it to the target location and then engage the target, giving three elementary mental operations. Rafal et al, ${ }^{5}$ using this paradigm, reported a

normal difference between "valid" and "invalid" conditions in PD patients with wearing-off phenomenon, and they concluded that these patients have no disturbance in shifting attention.

Recently, Posner et al ${ }^{6}$ reported a directional difference in shifts of attention and examined more complicated invalid conditions. They found that RTs to movements of the target inward "towards the fovea" are faster than movements outward "away from the fovea" in normal young subjects. By analysing the pattern of RTs involving a finger press of the same hand for all conditions, we can identify a disturbance in covert orienting attention in PD without considering any motor disturbance. This study attempted to analyse covert orienting attention in nondemented PD patients with either mild or moderate disabilities using the technique proposed by Posner. ${ }^{4}$ In our experiment, however, a slight modification was made to reduce error responses and to provide stronger focusing of attention during the precue period.

\section{Materials and methods \\ Subjects}

Twenty patients with idiopathic Parkinson's disease were studied. None of the patients had clinical evidence of impairment of intellectual function, and all were within normal range on the mini-mental state test scores. All were being treated with anti-Parkinsonian drugs. Medication was continued during the experiment. They were divided into two groups according to the grade of severity (Hoehn and Yahr). Ten patients (four males and six females) were grade I-II (P1) and had an age range of 49 to 66 years, mean (SD) 58.5 $(6 \cdot 3)$. Mean duration of illness was $4 \cdot 3$ years (3.1). The other ten patients (five males and five females) were grade III-IV (P2) and had an age range of 57 to 75 years, mean $68.7(5 \cdot 0)$ and a mean duration of illness of $10 \cdot 1$ years $(6 \cdot 8)$. Between the P1 and P2 groups there were significant differences in age $(t=3.81$, $\mathrm{p}<0.01)$ and in duration of illness $(t=2.45, \mathrm{p}<0.05)$. None of the patients showed wearing-off or on-off phenomena.

The control group (N) consisted of 20 volunteers who were drawn from a group of Parkinsonian patients' spouses. They were divided into two groups: 10 subjects under the age of $64(\mathrm{~N} 1)$ and 10 over the age of 65 (N2). In the N1 group (four males and six females), the subjects were aged $45-62$ years, mean $55 \cdot 3$ $(5 \cdot 3)$. In the $\mathrm{N} 2$ group (five males and five females), they were aged $65-80$ years, mean $69 \cdot 8(5 \cdot 2)$. The N1 group did not differ from in revised form 3 October 1989.

Accepted 17 November 1989 


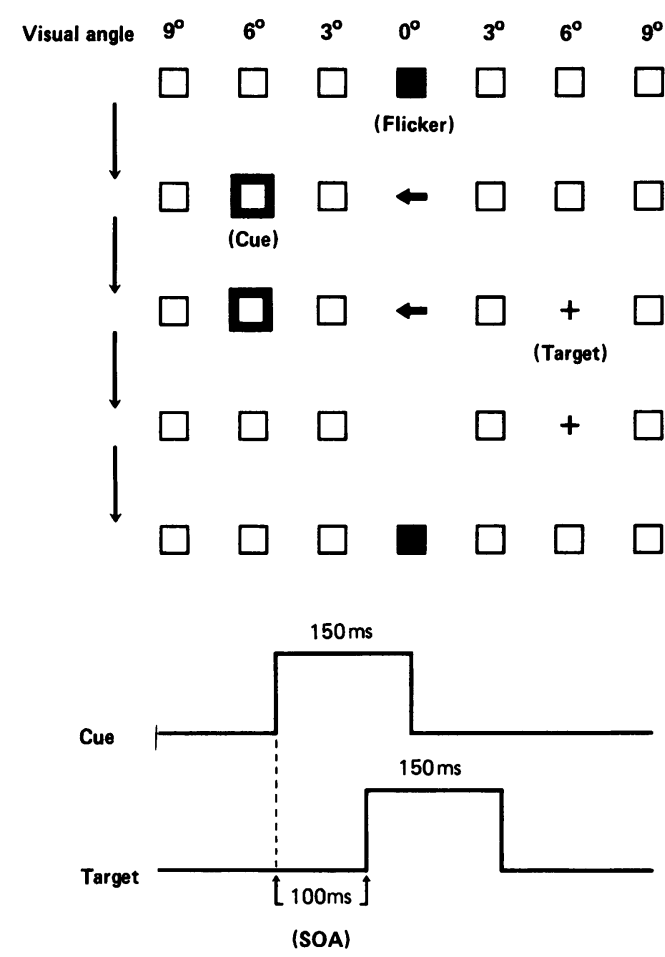

Figure 1 An example of the sequence of cue and target presentation in "crossed" conditions. Flicker of central square is followed by an arrow and a cue (brightening of the square). The subjects responded to a target (plus sign) with a right finger key press as quickly as possible. Stimulus onset asynchrony (SOA) was $100 \mathrm{~ms}$.

the P1 group for age $(t=1 \cdot 22)$ nor did the N2 group from the P2 group $(t=0 \cdot 96)$.

All the subjects were right handed. Informed consent was obtained from every subject.

\section{Methods}

Stimulus presentation and response recording was controlled using a computer (NEC, PC9801). The experiment was an RT task in which subjects were asked to press a single key as quickly as possible with the index finger of the right hand whenever a red cross (target) at one of the six positions in the visual field was presented. Before running the experiment, the subjects were instructed on the presentation of the cue which would precede the appearance of the target (fig 1 and 2). The subject sat in a comfortable chair in a quiet dark room and initially fixed on a central square occupying $1^{\circ}$ of visual angle and viewed one of eight colours, randomly presented in each trial. Three squares at $3^{\circ}, 6^{\circ}$ and $9^{\circ}$ on the left and three on the right side were presented on a colour display monitor (NEC, PC-551K). Each trial began with a flicker of the central square which lasted randomly between 250 and $2000 \mathrm{~ms}$. Subsequently, an arrow was presented in that centre square which indicated the direction (either right or left) of the appearance of a cue and thus reduced focusing of attention to a visual hemifield. At the same time the cue, which consisted of brightening one of the three squares on the indicated side, began and lasted $150 \mathrm{~ms}$. For "valid" conditions (fig 2, conditions 1-3) the target red cross then appeared in the same square as the cue, giving

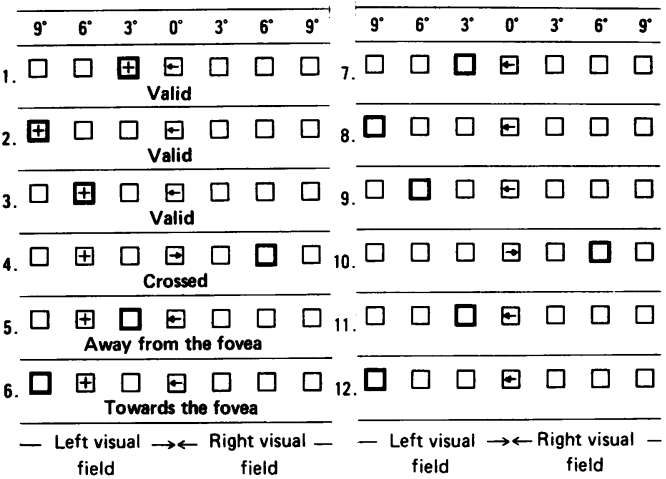

Figure 2 Cue and target (plus sign, only appearance in the left visual field) presentation in each condition examined. Conditions 1-6 show when the targets appear in the left visual field following a cue. Conditions 7-12 show when the cue was not followed by a target but otherwise correspond to the conditions presented as 1-6. The other 12 conditions, when the target appears in the right visual field, were also included in this experiment.

a total of six locations. For "invalid" conditions, the target occurred at $6^{\circ}$ following a cue appearing in a different square. Three types of "invalid" conditions in each visual field were used. In the "crossed" condition (fig 2, condition 4), the target was in the centre square on the side opposite the cue. The other two "invalid" conditions were "towards the fovea" (cue was at $9^{\circ}$ outside the target cue) and "away from the fovea" (cue was at $3^{\circ}$ inside the target) (fig 2, conditions 5-6). The target appeared $100 \mathrm{~ms}$ after the cue and persisted for $100 \mathrm{~ms}$ after it disappeared.

In this experiment, other trials used for excluding predictive responses of the subject, were those in which the cue was not followed by a target (fig 2, conditions 7-12). The number of such trials in each visual field was made equal to the number in which a target was prescribed, so that a total of 12 conditions in each visual field was used (fig 2). Ten trials in each condition giving a total of 240 trials, were done with each subject. Since the presentation of the target lasted $150 \mathrm{~ms}$ and the time from cue onset to target onset interval (stimulus onset asynchrony; SOA) was $100 \mathrm{~ms}$, the effect of the eye movement was negligible.

The data were subjected to analysis of variance (ANOVA) techniques. Since the variation in the observations correlated strongly with their level, an effective variancestabilising transformation (squared reciprocal, $1 / x^{2}$ ) was applied to the data for the purposes of analysis. Multiple post hoc comparisons were carried out using Duncan's method.

\section{Results}

RTs below $100 \mathrm{~ms}$ and above $1000 \mathrm{~ms}$ (accounting for less than $1 \%$ of the data) were excluded from the analysis. The means from the remaining trials were calculated for each subject in each condition, the means were calculated for the groups. In comparing results between the "valid" and "invalid" conditions, only data which were drawn from the target located at $6^{\circ}$ in each visual field were analysed for the valid condition.

Figure 3 shows the mean RTs for "valid", 


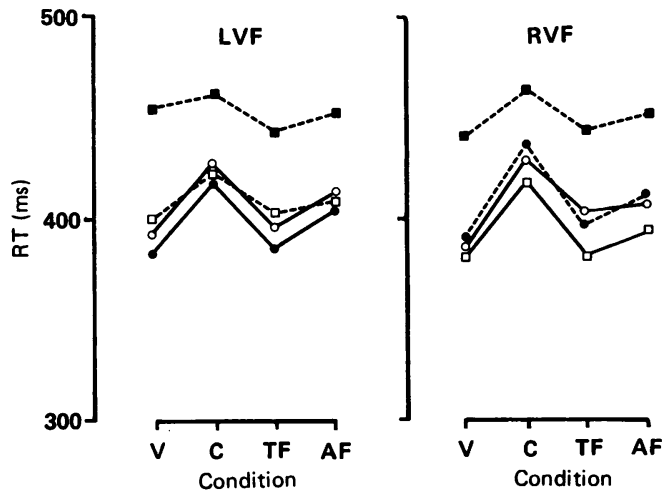

Figure 3 Mean reaction times (RTs) for four conditions in four groups. ?: N1 group, ?: N2 group, ?: P1 group, ?: P2 group. Abbreviations are: LVF, left visual field; $R V F$, right visual field; V, "valid" condition; $C$, "crossed" condition; TF, "towards the fovea" condition; $A F$, "away from the fovea" condition.

"crossed", "towards the fovea" and "away from the fovea" conditions for the four groups of subjects.

One way ANOVA comparing left and right visual fields in each condition showed no significant differences. The data were therefore collapsed across the visual fields, and a threeway analysis of variance was carried out, examining the effects of the two groups (controls and PD patients), the two age levels (young and old) and the four conditions ("valid", "crossed", "towards the fovea" and "away from the fovea"), as well as all levels of interactions. The analysis showed significant differences between patient groups $(\mathrm{p}<0.001$, control group mean (SD) $401.0 \mathrm{~ms}(50.3)$, patient group mean: $429.7 \mathrm{~ms}(81 \cdot 2)$, between age groups, ( $p<0.001$, young mean: $407.1 \mathrm{~ms}$ (54.5), old mean: $423.6 \mathrm{~ms}(80.2)$ ), and among conditions ( $p<0.001$, "valid" mean: $403.2 \mathrm{~ms}$ $(60.4))$, "crossed" mean: $434.6 \mathrm{~ms}$ (67.3), "towards fovea" mean: $406.6 \mathrm{~ms} \mathrm{(71.4),} \mathrm{"away}$ from fovea" mean $417.0 \mathrm{~ms}(72 \cdot 8)$ ), ordered statistically as: ("valid", "towards the fovea") < "away from the fovea" < "crossed".

No significant patient group-by-condition or age group-by-condition interactions were identified. Interactions of patient group-byage group ( $p<0.001)$ and the three-way interactions of patient group-by-age group-bycondition $(p<0.05)$ were significant. Post hoc comparisons of these interactions showed that the most prolonged RTs were in the P2 group (p < 0.05). Furthermore, the N1, N2 and P1 groups showed significant differences $(p<$ 0.05 ) in RT between "valid" and "crossed" conditions, whereas there was no such significant difference in the P2 group. Furthermore, the difference in RT between "valid" and "towards the fovea" or "away from the fovea" were significant in the control ( $p<$ 0.05 ), but not in the Parkinsonian groups. When comparing "towards the fovea" and "away from the fovea", faster RTs in "towards the fovea" were seen in the N2 group but those conditions yielded similar results in the other three groups.

\section{Discussion}

Generally, in normal healthy persons, target detection at the cued location is more efficient than target detection at other locations in the visual field. ${ }^{7}$ The paradigm for studying covert orienting attention used by Posner has previously shown that this greater efficiency is observed if the interval between cue and target presentation (SOA) is $50-150 \mathrm{~ms}^{8}{ }^{8}$ The current results in normal groups, using a $100 \mathrm{~ms}$ interval, is consistent with this evidence: clear shortening of RTs in valid conditions over invalid conditions was seen. Posner ${ }^{6}$ also found that movements inward "towards the fovea" are faster than movements "away from the fovea" and similar results were obtained by us from control group N2 but not from N1.

The main finding in this experiment was that the more disabled PD patients differed from controls and less severely affected PD cases in demonstrating no advantage for the "valid" condition over "invalid" conditions. No extinction-type RT pattern was found, as has been shown in patients with parietal damage who have an impaired ability to disengage attention from a current focus when a target occurs contralateral to the lesioned hemisphere. ${ }^{9}$

Rafal et al, ${ }^{5}$ using a very similar method but with only "valid" and "crossed" conditions, showed the normal advantage of the valid condition in six PD patients ranging from 3370 years and with wearing-off phenomenon, the advantage persisted whether the patients were under medication or not. The same result was shown in a second report by Rafal et al, ${ }^{10}$ in which eight Parkinsonian subjects ranging from 48-85 years were examined. However, in this latter report, there was no description of the severity of symptoms or length of illness in each patient. As with the patients of Rafal et al, our study shows an advantage in the "valid" condition over the "crossed" condition in the P1 group. However, a different pattern was obtained in the P2 group which was older, more disabled and had a longer duration of illness than the P1 group. This difference between our findings and those of Rafal et al is therefore probably due to the condition of the patients examined. As shown in our analysis, age has significant effects in such experiments. Moreover, PD patients are heterogenous in many respects, ${ }^{11}$ and so the differences in symptoms, duration of illness and/or the effect of anti-Parkinsonian drugs may also contribute to the discrepancy in results between the two PD groups.

Rafal et $a l^{10}$ also reported a smaller effect of validity in the vertical plane in patients with progressive supranuclear palsy (PSP), and concluded that an impairment of orienting to the cue for the vertical plane is found in PSP patients. Similar results in the horizontal plane have been reported in patients with frontal damage. ${ }^{12}$ In the visual field contralateral to the lesion, two such damaged patients had almost identical RTs in the "valid" and "crossed" conditions. In both our experiment and Posner's ${ }^{6}$ the comparison between valid and invalid conditions was done with the target 
always in the same square in either visual field but with the cue in various locations. Consequently, the result obtained here may suggest that the covert shift of attention from the centre to the cue is very weak or does not occur in the more disabled PD patients; instead they may only shift from the centre (initial focus) to the target. Three elementary mental operations have been proposed in the experiment of covert orienting of attention. ${ }^{4}$ The advantage of the "valid" condition can be explained by facilitation of target detection, following focusing on the cue. From this point of view, the smaller RT difference between "valid" and "invalid" conditions in the P2 group suggests a slow moving attention indicated by Rafal et $a l^{1012}$ in patients with PSP or frontal lobe damage. Even in the P1 group, the lack of advantage of the "valid" condition over "towards the fovea" or "away from the fovea", suggests a mild disturbance in shifting attention.

Pathological studies on PD patients have revealed considerable variability in the topography of brain lesions and in the extent of loss of various neurotransmitters. ${ }^{13}$ Our patients in the P2 group had a longer duration of illness than those in the P1 group, so that the pathological changes may be more widespread and severe. According to Rafal et al, ${ }^{10}$ replacement of dopamine did not affect orienting attention in PD patients with wearing-off phenomenon. Consequently, the dysfunction of other neurotransmitters or neuromodulators, especially noradrenaline or serotonin, which may be related to the occasional occurrence of disorders of mood, affect and cognition in PD, may also be responsible for the abnormal pattern found in this experiment.

Cognitive deficits similar to those following frontal lobe damage have been observed in both PD $^{14}{ }^{15}$ and PSP. ${ }^{15}$ In PSP, dysfunction of the frontal lobes has also been indicated by decreased glucose consumption, ${ }^{16}$ blood flow and oxygen utilisation ${ }^{17}$ in the frontal cortex; these decreases are not due to cortical lesions, since the cortex is considered to be intact, and, unlike the nigrostriatal dopaminergic system, the mesocortical dopaminergic system is intact. In PD, both the nigrostriatal and mesocortical dopaminergic systems are involved and the cognitive disturbances similar to those seen after frontal lobe damage have been attributed to dysfunction of the caudatecortical loops or direct dopamine depletion of the frontal lobe. ${ }^{14}$ The similarity between PD, PSP and frontal lobe damage from the results obtained from experiments on covert orienting attention, suggests that dysfunction of the frontal lobe may contribute to abnormalities in moving visual attention.

1 Stern Y, Mayeux R, Cote L. Reaction time and vigilance in Parkinson's disease. Arch Neurol 1984;41:1086-9.

2 Girroti F, Grassi MP, Carella F, et al. Possible involvement of attention processes in Parkinson's disease. In: Yahr MD, Bergmann KJ, eds. Parkinson's Disease. Advances in Neurology. New York: Raven Press, 1986:425-9.

3 Villardita C, Smirni P, Zappala G. Visual neglect in Parkinson's disease. Arch Neurol 1983;40:737-9.

4 Posner MI. Orienting of attention. Quart J Exp Psychol 1980;32:3-25.

5 Rafal RD, Posner MI, Walker JA, Friedrich FJ. Cognition and the basal ganglia. Brain 1984;107:1083-94

6 Posner MI, Walker JA, Friedrich FA, Rafal RD. How do the parietal lobes direct covert attention? Neuropsychologia $1987 ; 25: 135-45$.

7 Bashinski HS, Bachrach VR. Enhancement of perceptual sensitivity as the result of selectively attending to spatial locations. Percept Psychophys 1980;28:241-8.

8 Posner MI, Cohen Y. Components of attention. In: Bouma $\mathrm{XH}$, Bowhuis D, eds. Attention and Performance $X$. Lawrence Erbaum, Hillsdale, New Jersey, 1984:531-56.

9 Posner MI, Walker JA, Friedrich FJ, Rafal RD. Effects of parietal injury on covert orienting of attention. $J$ Neurosci
$1984 ; 4: 1863-74$.

10 Rafal RD, Posner MI, Friedman JH, Inhoff AW, Bernstein E. Orienting of visual attention in progressive supranuclear palsy. Brain 1988;111:267-80.

11 Zetusky WJ, Jankovic J, Pirozzolo FJ. The heterogeneity of Parkinson's disease. Neurology 1985;35:522-6.

12 Rafal RD. Frontal lobe lesions slow the movement of visual attention. Neurology 1987;37(suppl):127.

13 Hornykiewicz O, Kish SJ. Biochemical pathophysiology of Parkinson's disease. In: Yahr MD, Bergmann KJ, eds. Parkinson's Disease. Advances in Neurology. New York: Raven Press, 1986:19-34

14 Gotham AM, Brown RG, Marsden CD. Frontal cognitive function in patients with Parkinson's disease "on" and "off" levodopa. Brain 1988;111:299-321.

15 Pillon B, Dubois B, Lhermitte F, Agid Y. Heterogeneity of cognitive impairment in progressive supranuclear palsy, Parkinson's disease, and Alzheimer's disease. Neurology Parkinson's disease,

16 D'Antona R, Baron JC, Samson Y, Serdaru M, Viader F, Agid Y, Cambier J. Subcortical dementia: frontal cortex hypometabolism detected by positron tomography in patients with progressive supranuclear palsy. Brain 1985;108:785-9.

17 Leenders KL, Frackwiak RSJ, Lees AJ. Steel-RichardsonOlszewski syndrome. Brain 1988;111:615-30. 